\title{
Effect of HMGB1 and RAGE on brain injury and the protective mechanism of glycyrrhizin in intracranial-sinus occlusion followed by mechanical thrombectomy recanalization
}

\author{
SHU-WEN MU ${ }^{1 *}$, YUAN DANG $^{2 *}$, YA-CAO FAN $^{3}$, HAO ZHANG $^{4}$, JIAN-HE ZHANG ${ }^{4,5}$, \\ WEI WANG ${ }^{3}$, SHOU-SEN WANG ${ }^{1,4}$ and JIAN-JUN GU ${ }^{4,6}$
}

\begin{abstract}
Departments of ${ }^{1}$ Neurosurgery and ${ }^{2}$ Comparative Medicine, Dongfang Affiliated Hospital of Xiamen University
(900 Hospital of The Joint Logistics Team); ${ }^{3}$ Fuzong Clinical Medical College of Fujian Medical University; Departments of ${ }^{4}$ Neurosurgery and ${ }^{5}$ Radiology, 900 Hospital of The Joint Logistics Team, Fuzhou, Fujian 350025; ${ }^{6}$ Department of Neurosurgery, Zhengzhou University People's Hospital, Henan Provincial People's Hospital, Zhengzhou, Henan 450000, P.R. China
\end{abstract}

Received January 20, 2019; Accepted June 7, 2019

DOI: $10.3892 /$ ijmm.2019.4248

\begin{abstract}
The key to successful treatment of cerebral venous-sinus occlusion (CVO) is the rapid recanalization of the sinus following venous-sinus occlusion; however, rapid recanalization of the sinus may also cause secondary cerebral injury. The present study examined mechanical thrombectomy-related brain injury and the possible molecular mechanisms following CVO recanalization, and investigated the protective effect of glycyrrhizin (GL) in CVO recanalization. The cerebral venous sinus thrombosis (CVST) model was induced in rats using $40 \% \mathrm{FeCl}_{3}$. Mechanical thrombectomy was performed at $6 \mathrm{~h}$ post-thrombosis. GL was administered to rats following thromboembolism. Neurological function and brain water content were measured prior to sacrifice of the rats. Serum malondialdehyde, superoxide dismutase and nitric-oxide synthase concentrations were measured. The expression levels of high-mobility group box 1 (HMGB1) and receptor of advanced glycation end products (RAGE) and its downstream inflammatory mediators were measured in serum and brain
\end{abstract}

Correspondence to: Professor Jian-Jun Gu, Department of Neurosurgery, Zhengzhou University People's Hospital, Henan Provincial People's Hospital, 7 Weiwu Road, Zhengzhou, Henan 450000, P.R. China

E-mail: gujianjundt@163.com

Professor Shou-Sen Wang, Department of Neurosurgery, 900 Hospital of The Joint Logistics Team, 156 Xi'erhuanbei Road, Fuzhou, Fujian 350025, P.R. China

E-mail:wshsen@126.com

${ }^{*}$ Contributed equally

Key words: cerebral venous-sinus occlusion, mechanical thrombectomy, recanalization, brain injury, high-mobility group box 1 , glycyrrhizin tissues. Rapid CVO recanalization caused brain injury, and the brain parenchymal damage and neurological deficits caused by CVO were not completely restored following recanalization. Similarly, following rapid recanalization in the venous sinus, the expression levels of HMGB1 and RAGE were lower than those in the CVST group, but remained significantly higher than those of the sham group. The combination of mechanical thrombectomy and GL improved cerebral infarction and cerebral edema in rats, and inhibited the extracellular transport of HMGB1, and the expression of downstream inflammatory factors and oxidative-stress products. The administration of exogenous recombinant HMGB1 reversed the neural protective effects of GL. In conclusion, mechanical thrombectomy subsequent to CVO in rats can cause brain injury following recanalization. HMGB1 and RAGE promote inflammation in the process of brain injury following recanalization. GL has a relatively reliable neuroprotective effect on brain injury by inhibiting HMGB1 and its downstream inflammatory factors, and decreasing oxidative stress.

\section{Introduction}

Cerebral venous sinus thrombosis (CVST) accounts for $0.5-1.0 \%$ of strokes and is relatively more frequent in young adults $(1,2)$. The clinical manifestations of CVST lack specificity, with headaches as the only symptom in most patients. In severe cases, patients may experience focal neurological symptoms, seizures and even coma (3). At present, the pathogenesis of CVST remains poorly understood. In addition, the low rate of incidence and its atypical symptoms and signs often lead to misdiagnosis, which delays the initiation of treatment.

The key to successful CVST treatment is rapid recanalization of the venous sinus. Mechanical thrombectomy can rapidly restore blood flow in the sinus, which is an efficacious treatment for patients with progressive deterioration of nerve function. However, even with active venous-sinus mechanical thrombectomy, the prognosis of some patients with CVST is not improved (4). The reason for this may be similar to the 
pathophysiological mechanism of acute arterial occlusion clinically. Although stent thrombectomy can rapidly restore blood flow in a short time, blood-flow reperfusion further aggravates the inflammatory reaction and apoptosis caused by ischemia. These pathological changes comprise the primary mechanism of neuronal damage (5). Studies have found that HMGB1 serves an important role in brain ischemia/reperfusion (I/R) injury (6). Therefore, it was hypothesized that, due to the high rate of venous-sinus flow, rapid recanalization of an occluded sinus may occur similar to that in I/R injury following arterial occlusion, and that HMGB1 may serve an important role in this process.

Glycyrrhizin (GL) is a natural glycosyl-triterpenoid compound with anti-inflammatory and antitumor effects. Stronger Neo-Minophagen C (SNMC), with GL as its main component, has been used in the clinical treatment of chronic hepatitis (7). SNMC inhibits the release of HMGB1 from damaged cells by inhibiting the phosphorylation of HMGB1, and also inhibits the expression of HMGB1 $(8,9)$. Studies have shown that GL can improve cerebral infarction and cerebral edema following brain I/R injury by inhibiting HMGB1 and its downstream inflammatory signaling pathway $(10,11)$.

In the present study, based on successful establishment of a rat CVST model, the mechanical thrombectomy technique commonly used in clinical practice was simulated. The study examined possible neuronal damage and its associated molecular mechanisms subsequent to venous-sinus recanalization following mechanical thrombectomy, and examined the neuroprotective role of GL in CVO recanalization.

\section{Materials and methods}

Animals. The present study was approved by the Ethics Committee of Fuzhou General Hospital (Fuzhou, China). All animal experiments were conducted in accordance with the guidelines for the care and use of laboratory animals. A total of 132 male Sprague-Dawley rats (body weight 240-260 g, 6 weeks old) were provided by Shanghai SLAC Laboratory Animals Co., Ltd. (Shanghai, China). The rats were housed in a standard environment at $26-28^{\circ} \mathrm{C}$ under a 10 -h light/14-h dark light-dark cycle, and were provided with sufficient food and water.

Experimental animal grouping. In order to investigate the occurrence of brain injury following mechanical thrombectomy for CVO, 36 rats were randomly divided into three groups, with 12 rats in each of the following groups: Sham, CVST, and mechanical thrombectomy. To examine the protective effect of GL, 60 rats were randomly divided into five groups, with 12 rats in each of the following groups: Mechanical thrombectomy + normal saline group (NS), mechanical thrombectomy $+2 \mathrm{mg} / \mathrm{kg}$ GL group $(2 \mathrm{mg} / \mathrm{kg})$, mechanical thrombectomy $+4 \mathrm{mg} / \mathrm{kg}$ GL group ( $4 \mathrm{mg} / \mathrm{kg}$ ), mechanical thrombectomy $+10 \mathrm{mg} / \mathrm{kg}$ GL group $(10 \mathrm{mg} / \mathrm{kg})$, and mechanical thrombectomy + optimal-dose GL + recombinant (r)HMGB1 (100 mg per rat) group (GL + rHMGB1). In these groups, NS, GL or rHMGB1 (dissolved in saline; Asahi Kasei Pharma, Tokyo, Japan) were injected intraperitoneally $1 \mathrm{~h}$ before mechanical thrombectomy. To determine the effect of the time of administration on GL protection, 36 rats were randomly divided into three groups, with 12 rats in each of the following groups: $1 \mathrm{~h}$ before mechanical thrombectomy $(0 \mathrm{~h}), 6 \mathrm{~h}$ after mechanical thrombectomy $(6 \mathrm{~h})$, and $12 \mathrm{~h}$ after mechanical thrombectomy (12 h). In these groups, GL was injected in equal doses for each group.

Animal modeling. Rat sagittal-sinus thrombosis was induced using a $40 \% \mathrm{FeCl}_{3}$ solution (12). The rats were anesthetized with an intraperitoneal injection of sodium pentobarbital (40 mg/kg). The animals were placed on the operating table in the prone position to maintain horizontal position of the calvaria. The skin on the head was sterilized. Subsequently, $2 \%$ lidocaine $(0.1 \mathrm{ml})$ was used to pre-anesthetize the surgical incision. A cut at a length of $\sim 1.5 \mathrm{~cm}$ along the midline was made to carefully separate the muscles and periosteum, and to reveal the skull. A dental electric grinder was then used to drill between the bregma and lambda sutures under an operating microscope, and a bone window of $\sim 10 \mathrm{~mm}$ along the sagittal suture was opened. A filter strip of $40 \% \mathrm{FeCl}_{3}$ was placed on the superior sagittal sinus (SSS) for $5 \mathrm{~min}$, following which the residual $\mathrm{FeCl}_{3}$ was washed away with normal saline. Subsequently, the skin was sutured (size 0) and disinfected using iodophor. The animals were kept warm until awaking and were then placed in a single cage with access to food and water. At $6 \mathrm{~h}$ post-CVST, anesthesia was successfully performed. The rats were fixed at the prone position, the suture was cut, the bone window was washed with saline, and a blood-collection needle was used to gently puncture the sinus wall of the sagittal sinus at the leading edge of the bone window. A 0.014-inch nerve-intervention microfilament (Aashi Intecc, Nagoya, Japan) was inserted slowly into the venous sinus to perform thrombectomy. The guide wire was gently pushed $\sim 1 \mathrm{~cm}$ into the SSS and was then slowly pulled out, for a total of three times, to observe the venous flow in the venous sinus (Fig. 1A-C). Gelatin-sponge compression was used to stop bleeding, and normal saline was used to wash the wound. On confirming that there was no active bleeding, a size- 0 suture thread was used for suturing and the wound was disinfected using iodophor. The animals were kept warm until awake and were then placed in a single cage with access to food and water.

Neurological evaluation. Neurological evaluation was performed in a blinded-manner. Neurological evaluation of the rats was performed prior to surgery and $24 \mathrm{~h}$ following CVST with reference to a modified neurological severity score (mNSS) (13). All actions were scored separately by two researchers and the average scores were used for analysis. The mNSS mainly included the evaluation of movement, sensation, balance and reflexes of the rats. Normal rats were given a score of 0 . A higher score reflected more severe injury, and the maximum score was 18 points.

Staining with 2,3,5-triphenyltetrazolium chloride (TTC) and determination of cerebral infarction volume. The rats were sacrificed by intraperitoneal injection of an excess of sodium pentobarbital $24 \mathrm{~h}$ after CVST, and brain death (ceasing of self-breathing) was confirmed prior to decapitation and brain harvesting. The brain tissue was coronally sectioned at intervals of $2 \mathrm{~mm}$ and was then stained in a $1 \%$ TTC phosphate-buffer 

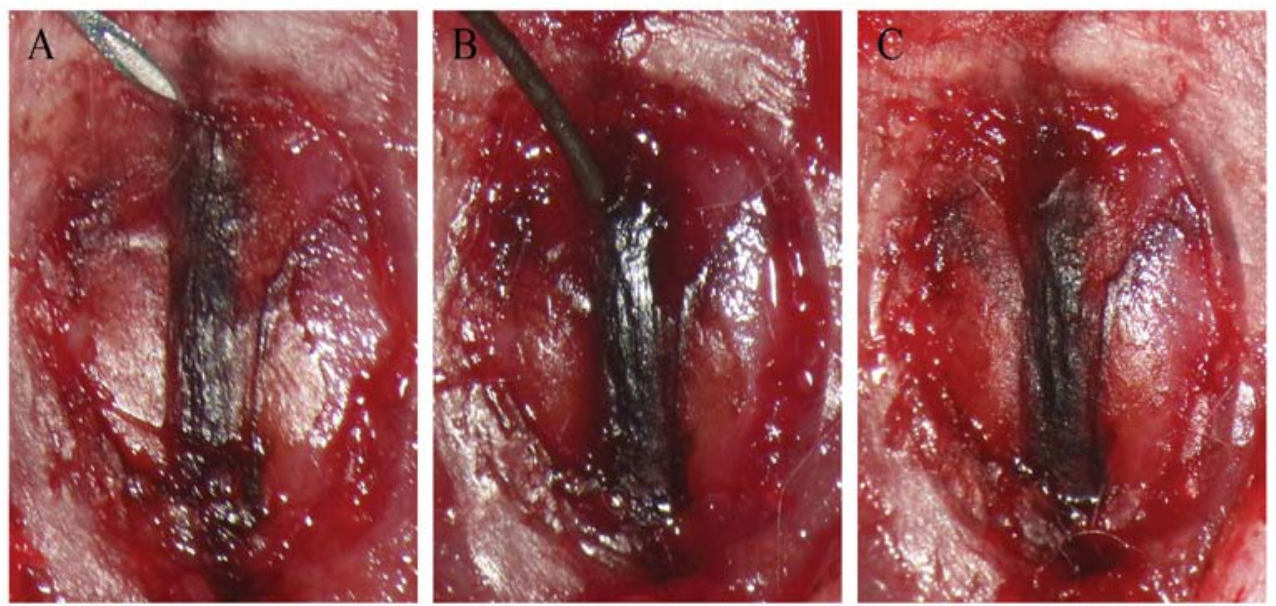

Figure 1. Process of thrombectomy. (A) A blood-collection needle pierces the superior-sagittal sinus vessel wall at the anterior bone window. (B) Slow insertion of a 0.014-inch micro-guide wire into the venous sinus for mechanical thrombectomy. (C) A gelatin sponge is pressed to stop bleeding following insertion.

solution (Nanjing Jiancheng Bioengineering Institute, Nanjing, China) at $37^{\circ} \mathrm{C}$ for $30 \mathrm{~min}$ in the dark. Following successful staining, the cells were fixed with a $10 \%$ paraformaldehyde solution for $10 \mathrm{~min}$. The sections were analyzed using the image-analysis software ImageJ version 1.44 (National Institutes of Health, Bethesda, MD, USA), and the infarct area, ipsilateral-hemisphere area and contralateral-hemisphere area of each section were measured. Each area was summed and multiplied by the section thickness $(2 \mathrm{~mm})$ to obtain the corresponding volume. In order to correct for the influence of ipsilateral-hemispheric edema on cerebral infarction, the relative infarct volume was calculated as follows (14): CIV $(\%)=[\mathrm{CHV}-(\mathrm{IHV}-\mathrm{IV})] \times 100 / \mathrm{CHV}$. CIV represents corrected infarct volume, $\mathrm{CHV}$ represents contralateral hemisphere volume, IHV represents ipsilateral hemisphere volume, and IV represents infarct volume.

Brain water content. Brain water content was determined using a dry- and wet-weight method (15). The rats were sacrificed by intraperitoneal injection of an overdose of sodium pentobarbital at $24 \mathrm{~h}$ after CVST. Following death, each rat was decapitated and $\sim 0.5 \mathrm{~g}$ of the cerebral cortical tissue of the sagittal sinus was removed and then weighed. The brain tissue was then placed in a clean, dry culture dish and heated in a hot oven at $110^{\circ} \mathrm{C}$ for $24 \mathrm{~h}$, following which the dry weight was weighed. The brain water content was calculated using the following formula: Brain water content $=[$ (wet weight-dry weight)/wet weight] x100\%.

Enzyme-linked immunosorbent assay (ELISA). The rats were sacrificed at the specified time points according to the experimental design, and blood samples $(5 \mathrm{ml})$ were collected from the abdominal aorta. The supernatant was extracted by centrifugation at $1,000 \mathrm{x}$ g for $10 \mathrm{~min}$ at $4^{\circ} \mathrm{C}$. Serum was stored in at $-20^{\circ} \mathrm{C}$ until ELISA analysis. Serum inflammatory factors, including HMGB1, RAGE, tumor necrosis factor- $\alpha$ (TNF- $\alpha$ ), interleukin-6 (IL-6) and IL-1 $\beta$, in addition to superoxide dismutase (SOD), nitric oxide synthase (NOS) and malondialdehyde (MDA), were quantified using specific ELISA kits according to the manufacturer's instructions (Nanjing Jiancheng Bioengineering Institute).
Reverse transcription-quantitative polymerase chain reaction $(R T-q P C R)$ analysis. Coronal sectioning was performed $\sim 2 \mathrm{~mm}$ to the left and right of the SSS. Subsequently, a cut was made along the upper edge of the hippocampus. The outer cortex was the penumbra and the medial cortex was the infarction core region. Total RNAs were isolated from the penumbra of the infarct hemisphere using TRIzol reagent (Invitrogen; Thermo Fisher Scientific Inc. Waltham, MA, USA) according to the manufacturer's instructions, and total RNAs were then reverse transcribed into cDNA using the High-Capacity cDNA RT kit (Roche Diagnostics GmbH, Mannheim, Germany) at $37^{\circ} \mathrm{C}$ for $15 \mathrm{~min}$. The mRNA expression levels of HMGB1 and RAGE were analyzed by qPCR using the SYBR Green PCR Master Mix kit (Applied Biosystems; Thermo Fisher Scientific Inc.) and the ABI-Prism 7300 system (Applied Biosystems; Thermo Fisher Scientific Inc.). The thermal cycling parameters were as follows: Heat-activated enzyme was activated by heating at $95^{\circ} \mathrm{C}$ for $10 \mathrm{~min}$, followed by 40 cycles of heating at $95^{\circ} \mathrm{C}$ for $15 \mathrm{sec}$ to denature, heating to $60^{\circ} \mathrm{C}$ for $30 \mathrm{sec}$ to anneal, and final elongation at $72^{\circ} \mathrm{C}$ for $30 \mathrm{sec}$. The concentration of the target genes was calculated by comparing the quantitative cycle $(\mathrm{Cq})$ value in each sample with the $\mathrm{Cq}$ value of the internal standard curve using the $2^{-\Delta \Delta \mathrm{Cq}}$ method (16). Melting-curve analysis and gel-electrophoresis evaluation of the RT-qPCR products were routinely performed to determine reaction specificity. GAPDH mRNA was set as an internal reference to quantify the level of targeted mRNA. The following primers were used: GAPDH, forward 5'-AGCAGG CTGACAGTGGAGTT-3', and reverse 5'-AGCAGGCTGACA GTGGAGTT-3'; RAGE, forward 5'-CGAGTCCGTGTCTAC CAGATT-3' and reverse 5'-GCGGCTGGAATGGAAACT GAA-3'; and HMGB1, forward 5'-GCTCCATAGAGACAG CGCCGGG-3' and reverse 5'-CCTCAGCGAGGCACAGAG TCGC-3'.

Western blotting. The dissected brain tissues from the areas of infarction were placed in RIPA buffer (Beyotime Institute of Biotechnology, Shanghai, China). The protein concentration was determined using a Bradford protein quantification kit (Bio-Rad Laboratories, Inc., Hercules, CA, USA). The protein-loading quantity was $40 \mu \mathrm{g}$ on an $8-15 \%$ SDS-PAGE 

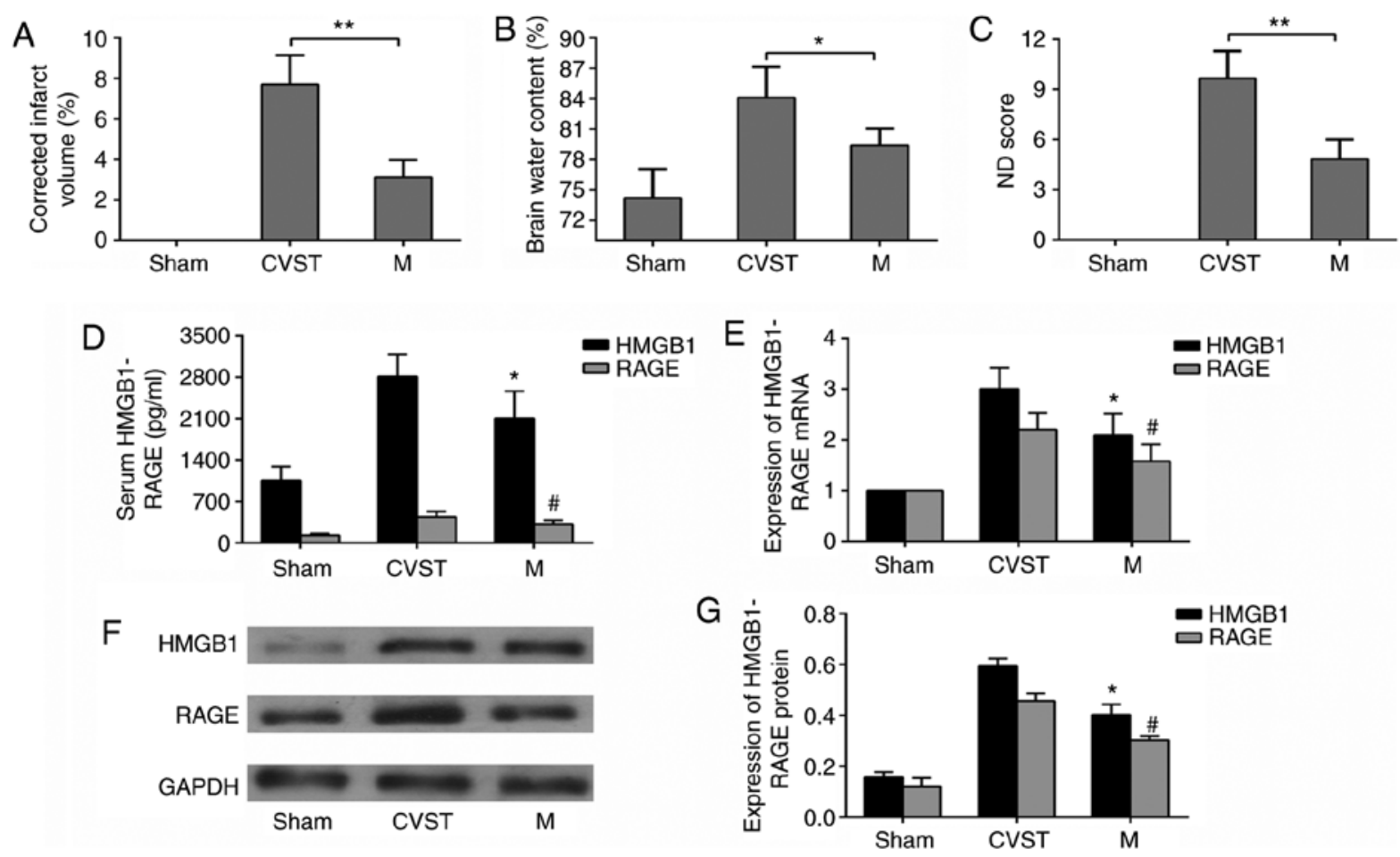

Figure 2. Changes in infarct volume, brain water content and ND scores following mechanical thrombectomy, and changes in mRNA and protein expression levels if HMGB1 and RAGE. (A) Quantification of 2,3,5-triphenyltetrazolium chloride staining results, (B) brain water content and (C) neurological function scores, showing that brain parenchymal damage improved following mechanical thrombectomy but was not completely reversed. " $\mathrm{P}<0.05$, ${ }^{* *} \mathrm{P}<0.01$. (D) ELISA results showing that serum concentrations of HMGB1 and RAGE decreased following mechanical thrombectomy. (E) Reverse transcription-quantitative polymerase chain reaction results showing that the mRNA expression levels of HMGB1 and RAGE in paranasal sinus brain tissues were downregulated following mechanical thrombectomy, (F) Western blot analysis and (G) quantitative analysis showing that HMGB1 and RAGE proteins were downregulated in paranasal sinus brain tissues following mechanical thrombectomy. ${ }^{*} \mathrm{P}<0.05$ and ${ }^{\#} \mathrm{P}<0.05$ vs. CVST group. Data are expressed as the mean \pm standard deviation. Intergroup differences were analyzed using Student's t-test or one-way analysis of variance. Tukey's post hoc test was used for multiple comparisons among various groups. HMGB1, high-mobility group box 1; RAGE, receptor of advanced glycation end products; CVST, cerebral venous sinus thrombosis; $\mathrm{M}$, mechanical thrombectomy; ND, neurological deficit.

gel. The protein bands were separated by electrophoresis and were electroporated onto a PVDF membrane. Following blocking with $5 \%$ skim milk in a Tris-buffered saline solution (with $1 \%$ Tween-20), the primary antibody was added and allowed to incubate overnight at $4^{\circ} \mathrm{C}$. The antibodies used were monoclonal rabbit anti-HMGB1 (1:1,000; cat. no. ab79823; Abcam, Cambridge, MA, USA) and monoclonal rabbit anti-RAGE (1:500; cat. no. ab3611; Abcam). The membranes were washed three times with PBS-Tween 20 and then incubated with horseradish peroxidase-labeled goat anti-rabbit IgG secondary antibody (1:500; cat. no. ab7090; Abcam) for $1 \mathrm{~h}$ at room temperature. Development was performed using the SuperSignal ${ }^{\mathrm{TM}}$ West Pico chemiluminescent substrate (Invitrogen; Thermo Fisher Scientific, Inc.). The internal reference used was $\beta$-actin (1:500; cat. no. ab8227; Abcam), and optical-density analysis was performed using ImageJ version 1.44 (National Institutes of Health).

Immunofluorescence. The rats were anesthetized with an intraperitoneal injection of sodium pentobarbital $24 \mathrm{~h}$ after CVST. Following successful anesthesia, $4 \%$ paraformaldehyde was used for fixation via cardiac perfusion. The signs of upper-extremity twitching and stiffness in the rats indicated adequate perfusion. Following perfusion using $100 \mathrm{ml}$ of the fixative, the rats were sacrificed and brain tissue was obtained and fixed in $4 \%$ paraformaldehyde for $24 \mathrm{~h}$. Coronal brain sections $(5-\mu \mathrm{m})$ from the ischemic core region were immunostained. The rabbit anti-HMGB1 antibody was used at a dilution of 1:200 (cat. no. ab79823; Abcam) overnight at $4^{\circ} \mathrm{C}$. 488 AffiniPure Fab Fragment Goat Anti-Rabbit IgG (1:200; cat. no. 111-547-003, Jackson ImmunoResearch Laboratories, West Grove, PA, USA) was used as a secondary antibody against HMGB1 for $1 \mathrm{~h}$ at $4^{\circ} \mathrm{C}$. The rabbit anti-RAGE antibody was used at a dilution of 1:200 (cat. no. ab3611; Abcam) overnight at $4^{\circ} \mathrm{C}$, and 594 AffiniPure Goat Anti-Rabbit IgG (1:500; cat. no. 111-585-003; Jackson ImmunoResearch Laboratories) was used as a secondary antibody against RAGE for $1 \mathrm{~h}$ at $4^{\circ} \mathrm{C}$. The sections were counterstained with $20 \mathrm{ml}$ DAPI (cat. no. 236275; Roche Diagnostics $\mathrm{GmbH}$ ) for $30 \mathrm{~min}$ at $4^{\circ} \mathrm{C}$ to stain the cell nucleus. The slides were coverslipped using glycerin and images were captured under a fluorescent microscope. Quantitative data were derived from the immunofluorescent images by pixel analyses using Image-Pro Plus 6.0 software (Media Cybernetics, Rockville, MD, USA).

Statistical analysis. Data are expressed as the mean \pm standard deviation. Statistical analyses were performed with SPSS 19.0 (IBM Corp., Armonk, NY, USA). Intergroup differences were analyzed using Student's t-test or one-way analysis of variance. Tukey's post hoc test was used for multiple comparisons among various groups. $\mathrm{P}<0.05$ was considered to indicate a statistically significant difference. 
A

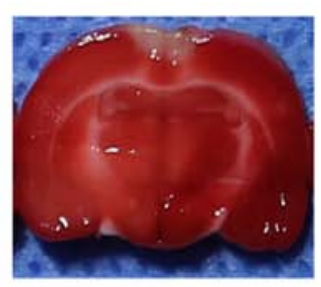

$\mathrm{B}$

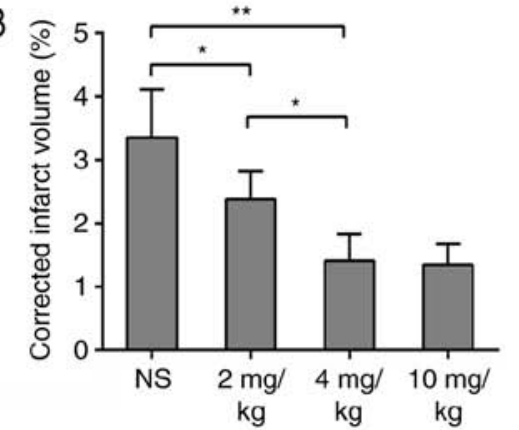

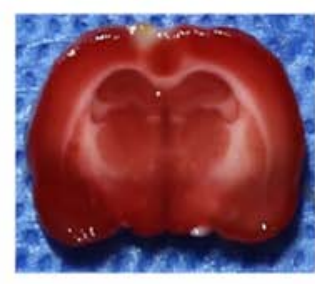
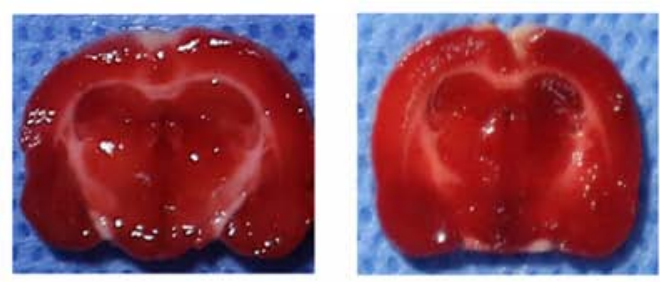

C

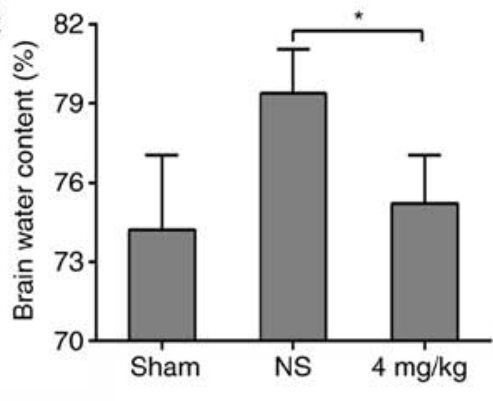

D

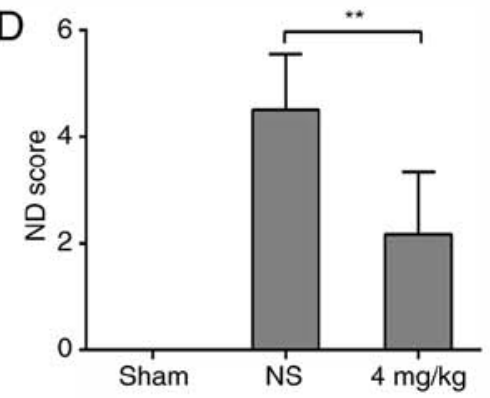

Figure 3. GL protection against venous ischemia/reperfusion injury. Different concentrations of GL were used to interfere with mechanical thrombectomy in rats. (A) 2,3,5-triphenyltetrazolium chloride staining was used to observe infarction and (B) image processing software quantified results. Different concentrations of GL reduced infarct volume. (C) Brain water content in the $4 \mathrm{mg} / \mathrm{kg}$ GL group was lower than that in the NS group. (D) Neurological function score in the $4 \mathrm{mg} / \mathrm{kg}$ GL group was lower than that in the NS group, and no neurological damage was present in the sham group. Data are expressed as the mean \pm standard deviation. Intergroup differences were analyzed using one-way analysis of variance. Tukey's post hoc test was used for multiple comparisons among various groups. ${ }^{*} \mathrm{P}<0.05,{ }^{* *} \mathrm{P}<0.01$. GL, glycyrrhizin; NS, normal saline; ND, neurological deficit.

\section{Results}

Brain injury following recanalization caused by mechanical thrombectomy for $\mathrm{CVO} . \mathrm{FeCl}_{3}$ was used to induce SSS occlusion in rats. To verify the stability of the model, TTC staining of a coronal section of the brain was performed. No injury was observed in the sham group, whereas a sinus cortex infarction area was observed in the CVST group. Mechanical thrombectomy was performed $6 \mathrm{~h}$ after CVST in the recanalization group. The infarct size was reduced compared with that in the CVST group, although it remained significantly different from that in the sham group (Fig. 2A). In addition, in terms of the neurological function scores and brain water content measurements of each group, mechanical thrombectomy reduced neurological deficits and cerebral edema, but did not restore them to the levels in the sham group (Fig. 2B and C). To determine whether HMGB1 and RAGE were involved in brain damage following recanalization for $\mathrm{CVO}, \mathrm{mRNA}$ and protein expression in serum and brain tissues were measured. As mRNA is more susceptible to degradation than protein, the detection of mRNA was selected in the penumbra, whereas the detection of protein was selected in the infarct area. The serum concentrations of HMGB1 and RAGE were lower than those in the CVST group following recanalization, but remained significantly higher than those in the sham operation group (Fig. 2D). Changes in the mRNA (Fig. 2E) and protein (Fig. 2F and G) expression of HMGB1 and RAGE in brain tissues showed the same trend. These results confirmed that recanalization-induced brain damage was present following CVO mechanical thrombectomy, and that HMGB1 and RAGE may be involved in this process.

GL protection against venous I/R injury. The CVST rats with mechanical thrombectomy were treated with intraperitoneal injection of different concentrations of GL. GL reduced infarct volume in a dose-dependent manner. The volumes of cerebral infarction in rats treated with 4 and $10 \mathrm{mg} / \mathrm{kg}$ GL were significantly decreased, and there was no significant difference between the two groups (Fig. 3A and B). To reduce the side effects of the drug, $4 \mathrm{mg} / \mathrm{kg}$ GL was selected for further experiments. In addition, the brain water content and neurological function scores of the $4 \mathrm{mg} / \mathrm{kg}$ GL-treated rats were significantly improved, to levels similar to those of the sham group (Fig. 3C and D). These data indicate that GL has a neuroprotective effect on brain damage following sinus recanalization.

GL inhibits the transfer of $H M G B 1$ and the expression of RAGE following recanalization. HMGB1 is located in the nucleus under physiological conditions (17). Using fluorescent staining, HMGB1 was observed in the nucleus of the sinus paracortex in the sham group. The expression of cytoplasmic and extracellular HMGB1 was significantly increased following mechanical thrombectomy, although some HMGB1 remained in the nucleus. Following GL treatment, it was identified that the level of cytosolic HMGB1 was significantly reduced compared with that in the simple mechanical thrombectomy group, and HMGB1 was increased in the nucleus (Fig. 4A and B). RAGE is an HMGB1 receptor located on the plasma membrane (18). A small amount of RAGE was expressed on the plasma membrane in the sham group. The expression of RAGE was significantly increased following CVST, and its expression was significantly decreased following further treatment with GL (Fig. 4C and D).

GL inhibits the expression of $H M G B 1$ and its downstream inflammatory factors following recanalization. Intervention with equal doses of GL was performed at different time points following mechanical thrombectomy in the rats. It was 
A
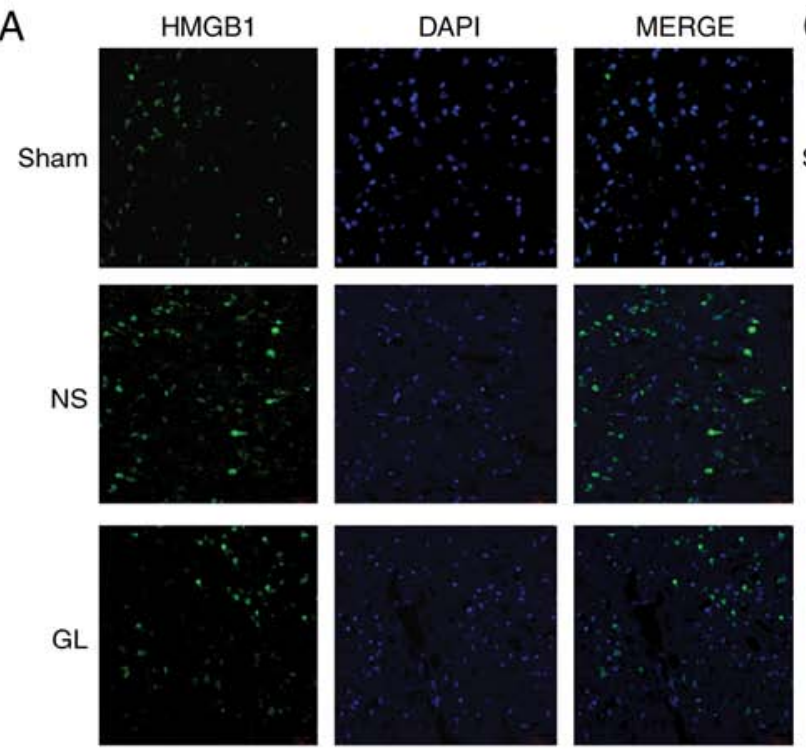

B

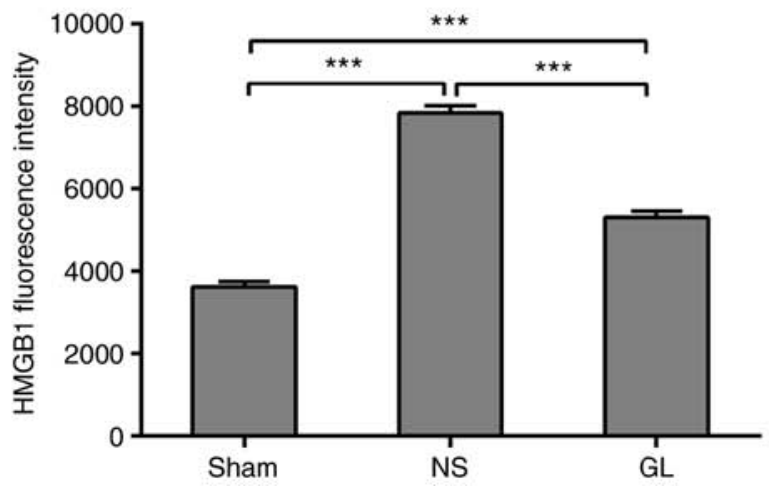

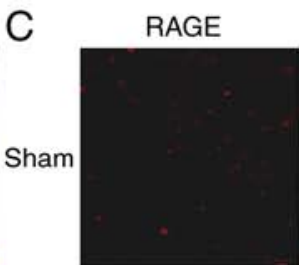
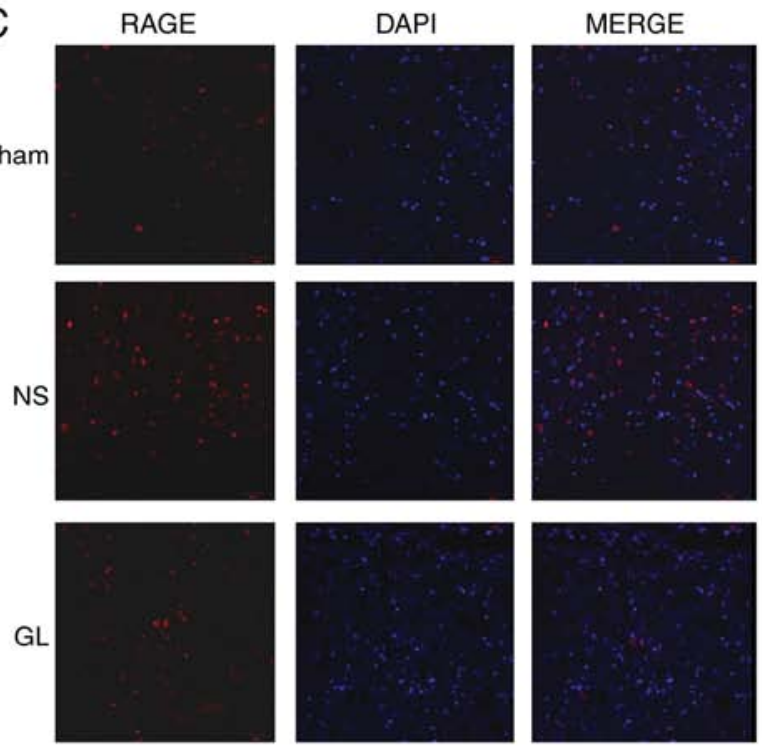

D

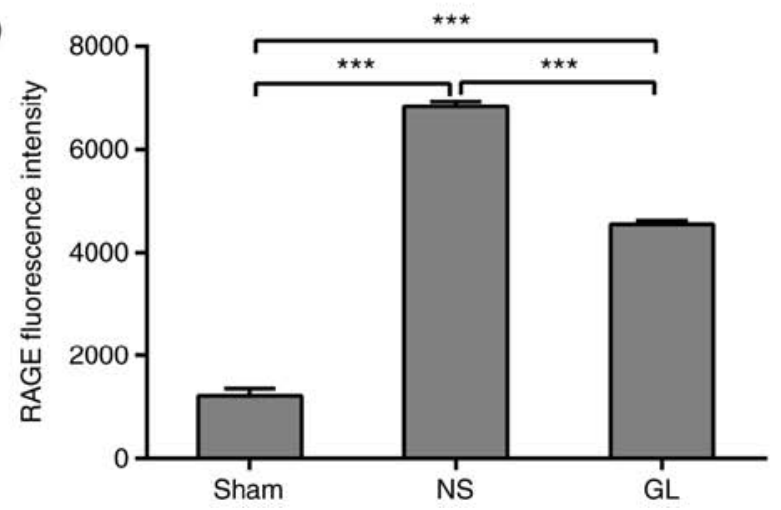

Figure 4. GL inhibits HMGB1 transport and the expression of RAGE following recanalization. (A) Immunofluorescent staining showing differences in the extracellular and intracellular distribution of HMGB1 in paranasal sinus brain tissues. Magnification, x200. (B) Quantification of HMGB1 fluorescence intensity. (C) Immunofluorescent staining showing changes in the expression of RAGE in paranasal sinus brain tissues. Magnification, x200. (D) Quantification of RAGE fluorescence intensity. ${ }^{* * *} \mathrm{P}<0.001$. GL, glycyrrhizin; NS, normal saline; HMGB1, high-mobility group box 1; RAGE, receptor of advanced glycation end products.

observed that GL injection $1 \mathrm{~h}$ before mechanical thrombectomy significantly reduced the concentrations of HMGB1 and RAGE in the serum compared with levels at 6 and $12 \mathrm{~h}$ post-thrombectomy (Fig. 5A). In order to investigate the source of HMGB1 and RAGE in the serum, the mRNA and protein expression levels of HMGB1 and RAGE in paranasal sinus tissues were measured by RT-qPCR and western blot analyses, respectively. It was found that GL injection $1 \mathrm{~h}$ before mechanical thrombectomy significantly inhibited the mRNA and protein expression of HMGB1 and RAGE. Injections at different time points led to a similar trend of change in serum concentrations (Fig. 5B-D). To investigate the effect of GL on the HMGB1-RAGE pathway, serum concentrations of TNF- $\alpha$, IL- $1 \beta$ and IL- 6 were measured by ELISA. The concentrations of TNF- $\alpha$, IL- $1 \beta$ and IL- 6 were significantly decreased in the $0 \mathrm{~h}$ group (Fig. 5E). The concentrations of MDA, SOD and NOS in the serum were measured using the same method, and the results followed a similar trend (Fig. 5F-H).

GL protects against brain damage following CVOrecanalization by antagonizing $H M G B 1$. In order to further confirm that GL protected brain damage following $\mathrm{CVO}$ recanalization by antagonizing HMGB1, GL was combined with rHMGB1. The resulting infarct volume was significantly increased compared with that of GL alone, with no significant difference compared with that of the NS group ( $\mathrm{P}>0.05$; Fig. 6A). Furthermore, changes in the concentration of HMGB1 and its downstream inflammatory factors were examined in the serum. Of note, the inflammatory index of the GL + rHMGB1 group was significantly increased compared with that of the GL-treated group (Fig. 6B and C). The same trend was found for oxidative stress-related indicators (Fig. 6D-F). These results further demonstrated that GL protects against brain damage following recanalization by inhibiting the HMGB1-RAGE pathway.

\section{Discussion}

In the present study, $\mathrm{FeCl}_{3}$ was used to induce CVST in rats, and mechanical thrombectomy was performed $6 \mathrm{~h}$ after thrombosis to simulate the clinical recanalization observed in human patients with CVST. The results demonstrated that CVST mechanical thrombectomy reduced the volume of cerebral infarction and reduced neurological deficits to a certain extent. However, this protective effect on cerebral infarction 

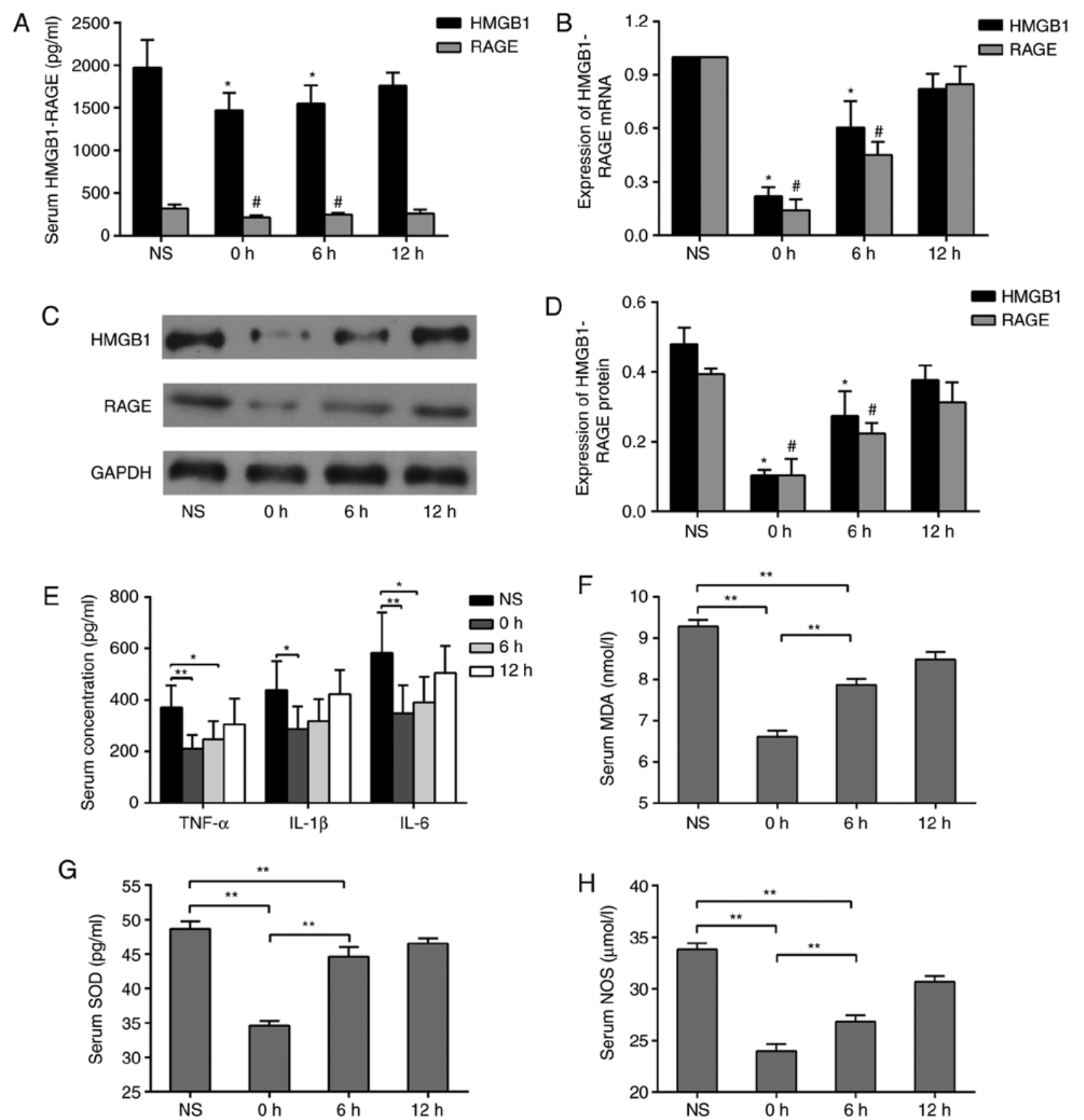

Figure 5. Expression of HMGB1 and its downstream inflammatory factors following GL inhibition. (A) Changes in serum concentrations of HMGB1 and RAGE at different administration time points. (B) Changes in mRNA expression levels of HMGB1 and RAGE in paranasal sinus brain tissues at different administration time points; (C) Changes in protein expression levels of HMGB1 and RAGE in sinus paraventricular tissues at different administration time points; (D) quantification of protein expression levels. ${ }^{*} \mathrm{P}<0.05$ and ${ }^{\#} \mathrm{P}<0.05$ vs. NS groups. Changes in serum concentrations of (E) TNF- $\alpha$, IL-1 $\beta$, IL-6, (F) MDA, (G) SOD and (H) NOS at different doses. ${ }^{*} \mathrm{P}<0.05,{ }^{* *} \mathrm{P}<0.01$. Data are expressed as the mean \pm standard deviation. Intergroup differences were analyzed using one-way analysis of variance Tukey's post hoc test was used for multiple comparisons among various groups. GL, glycyrrhizin; NS, normal saline; HMGB1, high-mobility group box 1; RAGE, receptor of advanced glycation end products; TNF- $\alpha$, tumor necrosis factor- $\alpha$; IL, interleukin; MDA, malondialdehyde; SOD, superoxide dismutase; NOS, nitric oxide synthase.

volume reduction and neurological function was limited; postoperative neurological function remained significantly different from that of the sham-operation group. In order to further improve neurological function, GL treatment was administered following mechanical thrombectomy. This combinatorial treatment was able to inhibit the extracellular transport of HMGB1, and inhibited the cascade of amplification of the HMGB1-RAGE inflammatory pathway and its downstream inflammatory factors.
Clinically, the main goal of treating acute ischemic stroke is to restore blood flow to ischemic brain tissue as soon as possible. Although this treatment can restore cerebral blood flow in a short time, improvements in cerebral infarction and nerve function are limited. This treatment may lead to more widespread damage of brain tissue at the ischemic penumbra, which is a phenomenon known as brain I/R injury (19). In this process, reperfusion of oxygen-carrying blood may further aggravate the inflammatory reaction and the oxidative stress 
A
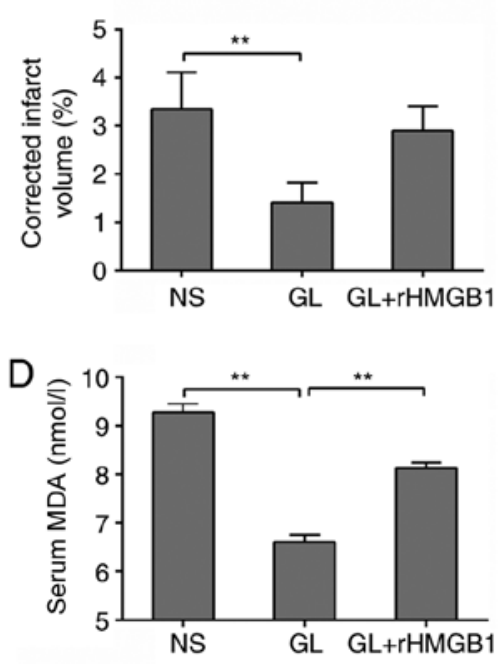

B

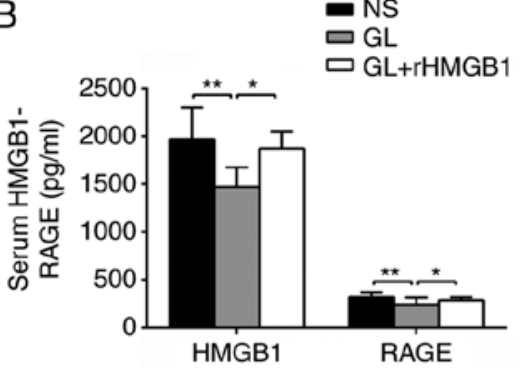

E

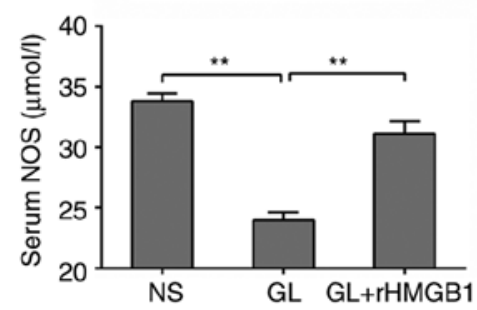

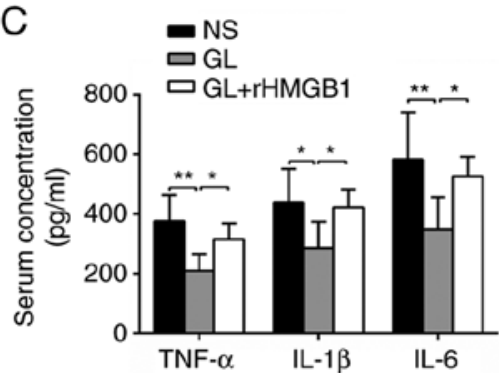

$\mathrm{F}$

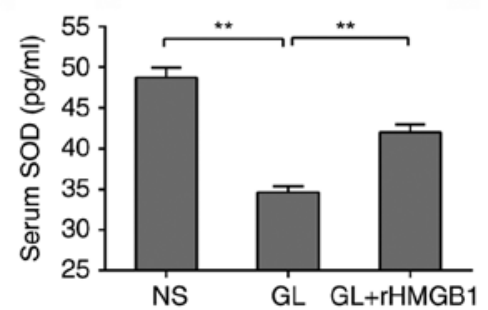

Figure 6. GL protects against brain damage following CVO recanalization by antagonizing HMGB1. (A) Changes in cerebral infarction volume in rHMGB1-treated or untreated rats. Changes in (B) HMGB1 and RAGE, (C) TNF- $\alpha$, IL-1 $\beta$, IL-6, (D) MDA, (E) NOS and (F) SOD in rHMGB1-treated or untreated rats. Data are expressed as the mean \pm standard deviation. Intergroup differences were analyzed using one-way analysis of variance. Tukey's post hoc test was used for multiple comparisons among various groups. ${ }^{*} \mathrm{P}<0.05,{ }^{* *} \mathrm{P}<0.01$. rHMGB1, recombinant high mobility group box 1 ; RAGE, receptor for advanced glycation end products; NS, normal saline; GL, glycyrrhizin; TNF- $\alpha$, tumor necrosis factor- $\alpha$; IL, interleukin; MDA, malondialdehyde; SOD, superoxide dismutase; NOS, nitric oxide synthase.

response of cerebrovascular endothelial cells caused by ischemia and hypoxia, which may further lead to destruction via cerebral infarction, damage to the blood-brain barrier and worsening of neural function (20). An increasing number of clinical cases indicates that this treatment has limitations $(5,21,22)$. As the SSS is the main reflux pathway of venous blood, long-term hypoxia and blood stasis can lead to cerebral edema and even infarction following SSS occlusion. Our previous studies and the results of the present study demonstrate that $\mathrm{FeCl}_{3}$-induced CVST in rats can cause parasitic brain tissue infarction, brain edema and other substantial damage (16). Therefore, a mechanism similar to that of arterial I/R was suggested, in which brain damage following recanalization occurs when the venous sinus is opened in brain tissues with substantial damage. However, in the present study, rat cerebral infarction and cerebral edema were marginally improved following mechanical thrombectomy, which differs from arterial I/R. The reason for this may be that the venous blood oxygen content is lower than that of the artery. Correspondingly, endothelial cells are also less susceptible to inflammatory and oxidative stress responses. However, the results of these experiments suggest that mechanical thrombectomy has limited improvement on brain damage and neurological function, and I/R injury may still occur. According to a recent meta-analysis, $40.2 \%$ of patients with CVST who underwent endovascular treatment developed brain injury or led to a coma, and the mortality rate was $14.3 \%$ at a later follow-up (4). Therefore, mechanical thrombectomy can lead to certain defects, which further confirms our hypothesis.

HMGB1 is a member of the damage-associated molecular pattern molecular family and is involved in the inflammatory cascade-amplification reaction in the pathophysiology of sepsis, tumors and brain trauma. Under normal physiological conditions, HMGB1 is expressed almost entirely in the nucleus of all cells and is encoded by the 13q12.3 gene (17). HMGB1 is essential for the maintenance of chromosomal structure and the physiological activities of DNA (23). Intracellular HMGB1 has the following two modes of release: Immediate release upon cell death, and active release under the action of stimulating factors (24). Under the action of inflammatory stimuli, HMGB1 released extracellularly can provide information to alert adjacent cells and activate the innate immune response. In addition, extracellular HMGB1 can induce endocytosis in combination with RAGE and carry other extracellular pro-inflammatory factors to lysosomes to transmit hazardous information in a timely manner (24). Inflammation is known to be important in arterial I/R injury (25). Kim et al showed that necrotic neurons in ischemic brain tissue can release HMGB1 in large quantities and serve a role in the formation of brain I/R injury (6). In our previous studies, the mRNA and protein expression levels of HMGB1 and RAGE were significantly upregulated in CVST rats with cerebral infarction (16). The inhibition of HMGB1 and RAGE by drugs significantly reduces nerve damage and reduces cerebral infarction volume.

GL has natural anti-inflammatory properties and has an antagonistic effect on HMGB1 (26). Ieong et al found that the use of GL significantly reduced HMGB1-positive cells, downregulated the mRNA and protein levels of HMGB1, and reduced brain-parenchymal damage following subarachnoid hemorrhage (27). The present study found that, following mechanical thrombectomy, a large amount of HMGB1 was transferred from the nucleus to the cytoplasm and was released to the outside of the cell, which may be the main reason for the increase of serum HMGB1 concentrations. The application of GL significantly suppressed the above-mentioned process. MDA, SOD and NOS are sensitive indicators for evaluating oxidative stress. These factors are activated following acute 
ischemic or hypoxic injury in the brain, which triggers inflammation and apoptosis and induces a series of brain injuries, including blood-brain-barrier destruction(28-30). In the present study, it was demonstrated that GL inhibited the expression of HMGB1 and RAGE and their downstream inflammatory factors following thrombectomy, and inhibited the expression of MDA, SOD and NOS. Following the combined administration of exogenous rHMGB1, the factors downregulated by GL protection were reversed to varying degrees. It may be that microglia are activated immediately following cerebral ischemia, and that HMGB1 activates microglia through the RAGE receptor on the plasma membrane, releasing pro-inflammatory factors including TNF- $\alpha$, IL- $1 \beta$ and IL- 6 and activating oxidative stress responses. Therefore, GL may have a neuroprotective effect on the activation of microglia by inhibiting HMGB1 during CVO thrombectomy.

Combined anti-inflammatory medications and intravascular management have gradually gained attention in clinical practice. The present study primarily confirmed recanalization-induced brain injury following CVO recanalization, and showed that HMGB1 and RAGE induced an inflammatory response in this process, whereas GL inhibited this signaling pathway by exerting neuroprotective effects in CVO recanalization. Considering inter-species differences between rats and humans, it is unclear whether the results of the present study can be extended to humans. Therefore, further translational research is required. In conclusion, the present study provides a solid basis for the clinical use of CVO mechanical thrombectomy in combination with neuroprotective agents to improve therapeutic outcomes.

\section{Acknowledgements}

The authors sincerely thank Dr Xian-hua Liu from Fuzhou Provincial Hospital for her capable technical assistance.

\section{Funding}

This study was supported by the Natural Science Foundation of Fujian Province Grant (grant no. 2017J01323) to Professor Jian-Jun Gu.

\section{Availability of data and materials}

The datasets used during the current study are available from the corresponding author on reasonable request.

\section{Authors' contributions}

SWM, YD, JJG and SSW were responsible for the study concept and design. SWM, YD, YCF and HZ performed the experiments. SWM, YD, JHZ and WW were responsible for data analyses and interpretations. SWM and JJG drafted the manuscript. All authors critically reviewed the manuscript and approved the final version.

\section{Ethics approval and consent to participate}

The present study was approved by the Ethics Committee of Fuzhou General Hospital. All animal experiments were conducted in accordance with the guidelines for the care and use of laboratory animals.

\section{Patient consent for publication}

Not applicable.

\section{Competing interests}

The authors declare that they have no competing interests.

\section{References}

1. Bousser MG and Ferro JM: Cerebral venous thrombosis: An update. Lancet Neurol 6: 162-170, 2007.

2. Stam J: Thrombosis of the cerebral veins and sinuses. N Engl J Med 352: 1791-1798, 2005.

3. Einhäupl K, Stam J, Bousser MG, De Bruijn SF, Ferro JM, Martinelli I and Masuhr F; European Federation of Neurological Societies: EFNS guideline on the treatment of cerebral venous and sinus thrombosis in adult patients. Eur J Neurol 17: 1229-1235, 2010.

4. Ilyas A, Chen CJ, Raper DM, Ding D, Buell T, Mastorakos P and Liu KC: Endovascular mechanical thrombectomy for cerebral venous sinus thrombosis: A systematic review. J Neurointerv Surg 9: 1086-1092, 2017.

5. van den Berg LA, Dijkgraaf MG, Berkhemer OA, Fransen PS, Beumer D, Lingsma HF, Majoie CB, Dippel DW, van der Lugt A, van Oostenbrugge RJ, et al: Two-year outcome after endovascular treatment for acute ischemic stroke. N Engl J Med 376: 1341-1349, 2017.

6. Kim ID, Shin JH, Kim SW, Choi S, Ahn J, Han PL, Park JS and Lee JK: Intranasal delivery of HMGB1 siRNA confers target gene knockdown and robust neuroprotection in the postischemic brain. Mol Ther 20: 829-839, 2012.

7. Manns MP, Wedemeyer H, Singer A, Khomutjanskaja N, Dienes HP, Roskams T, Goldin R, Hehnke U and Inoue H; European SNMC Study Group: Glycyrrhizin in patients who failed previous interferon alpha-based therapies: Biochemical and histological effects after 52 weeks. J Viral Hepat 19: 537-546, 2012.

8. Kim SW, Jin Y, Shin JH, Kim ID, Lee HK, Park S, Han PL and Lee JK: Glycyrrhizic acid affords robust neuroprotection in the postischemic brain via anti-inflammatory effect by inhibiting HMGB1 phosphorylation and secretion. Neurobiol Dis 46: 147-156, 2012.

9. Wu CX, He LX, Guo H, Tian XX, Liu Q and Sun H: Inhibition effect of glycyrrhizin in lipopolysaccharide-induced high-mobility group box 1 releasing and expression from RAW264.7 cells. Shock 43: 412-421, 2015.

10. Zhang J, Wu Y, Weng Z, Zhou T, Feng T and Lin Y: Glycyrrhizin protects brain against ischemia-reperfusion injury in mice through HMGB1-TLR4-IL-17A signaling pathway. Brain Res 1582: 176-186, 2014.

11. Gong G, Xiang L, Yuan L, Hu L, Wu W, Cai L, Yin L and Dong H: Protective effect of glycyrrhizin, a direct HMGB1 inhibitor, on focal cerebral ischemia/reperfusion-induced inflammation, oxidative stress, and apoptosis in rats. PLoS One 9: e89450, 2014.

12. Srivastava AK, Gupta RK, Haris M, Ray M, Kalita J and Misra UK: Cerebral venous sinus thrombosis: Developing an experimental model. J Neurosci Methods 161: 220-222, 2007.

13. Chen J, Li Y, Wang L, Zhang Z, Lu D, Lu M and Chopp M: Therapeutic benefit of intravenous administration of bone marrow stromal cells after cerebral ischemia in rats. Stroke 32: 1005-1011, 2001.

14. Ansari S, Azari H, McConnell DJ, Afzal A and Mocco J: Intraluminal middle cerebral artery occlusion (MCAO) model for ischemic stroke with laser doppler flowmetry guidance in mice. J Vis Exp: pii 2879, 2011.

15. Chen C, Wang Q, Gao Y, Lu Z, Cui X, Zheng T, Liu Y, Li X, He X, Zhang X, et al: Photothrombosis combined with thrombin injection establishes a rat model of cerebral venous sinus thrombosis. Neuroscience 306: 39-49, 2015.

16. Gu JJ, Chen JB, Zhang JH, Zhang $\mathrm{H}$ and Wang SS: Recombinant human soluble thrombomodulin protects against brain injury in a CVST rat model, via downregulation of the HMGB1-RAGE axis. Mol Med Rep 14: 5217-5222, 2016. 
17. Sohun M and Shen H: The implication and potential applications of high-mobility group box 1 protein in breast cancer. Ann Transl Med 4: 217, 2016.

18. Anggayasti WL, Mancera RL, Bottomley S and Helmerhorst E: The self-association of HMGB1 and its possible role in the binding to DNA and cell membrane receptors. FEBS Lett 591: 282-294, 2017.

19. Schaller B and Graf R: Cerebral ischemia and reperfusion: The pathophysiologic concept as a basis for clinical therapy. J Cereb Blood Flow Metab 24: 351-371, 2004.

20. Goyal M, Menon BK, van Zwam WH, Dippel DW, Mitchell PJ, Demchuk AM, Dávalos A, Majoie CB, van der Lugt A, de Miquel MA, et al: Endovascular thrombectomy after large-vessel ischaemic stroke: A meta-analysis of individual patient data from five randomised trials. Lancet 387: 1723-1731, 2016.

21. Alegiani AC, Dorn F, Herzberg M, Wollenweber FA, Kellert L, Siebert E, Nolte $\mathrm{CH}$, von Rennenberg R, Hattingen E, Petzold GC, et al: Systematic evaluation of stroke thrombectomy in clinical practice: The German stroke registry endovascular treatment. Int J Stroke: Oct 22, 2018 (Epub ahead of print).

22. Mizuma A and Yenari MA: Anti-inflammatory targets for the treatment of reperfusion injury in stroke. Front Neurol 8: 467, 2017.

23. Bertheloot D and Latz E: HMGB1, IL-1alpha, IL-33 and S100 proteins: Dual-function alarmins. Cell Mol Immunol 14: 43-64, 2017.

24. Andersson U, Yang $\mathrm{H}$ and Harris $\mathrm{H}$ : High-mobility group box 1 protein (HMGB1) operates as an alarmin outside as well as inside cells. Semin Immunol 38: 40-48, 2018.
25. Bai J and Lyden PD: Revisiting cerebral postischemic reperfusion injury: New insights in understanding reperfusion failure, hemorrhage, and edema. Int J Stroke 10: 143-152, 2015.

26. Mollica L, De Marchis F, Spitaleri A, Dallacosta C, Pennacchini D, Zamai M, Agresti A, Trisciuoglio L, Musco G and Bianchi ME: Glycyrrhizin binds to high-mobility group box 1 protein and inhibits its cytokine activities. Chem Biol 14: 431-441, 2007.

27. Ieong C, Sun H, Wang Q and Ma J: Glycyrrhizin suppresses the expressions of HMGB1 and ameliorates inflammative effect after acute subarachnoid hemorrhage in rat model. J Clin Neurosci 47: 278-284, 2018.

28. Jadhav RS, Ahmed L, Swamy PL and Sanaullah S: Neuroprotective effects of polyhydroxy pregnane glycoside isolated from Wattakaka volubilis (L.f.) Stapf. after middle cerebral artery occlusion and reperfusion in rats. Brain Res 1515: 78-87, 2013.

29. Zheng YQ, Liu JX, Wang JN and Xu L: Effects of crocin on reperfusion-induced oxidative/nitrative injury to cerebral microvessels after global cerebral ischemia. Brain Res 1138: 86-94, 2007.

30. Liu H, Li J, Zhao F, Wang H, Qu Y and Mu D: Nitric oxide synthase in hypoxic or ischemic brain injury. Rev Neurosci 26: $105-117,2015$.

This work is licensed under a Creative Commons Attribution-NonCommercial-NoDerivatives 4.0 International (CC BY-NC-ND 4.0) License. 